\title{
Phase-shift based BOTDA measurements tolerant to non-local effects
}

\author{
Javier Urricelqui*, Mikel Sagues, Alayn Loayssa \\ Departamento de Ingeniería Eléctrica y Electrónica, Universidad Pública de Navarra, \\ Campus Arrosadía s/n, 31006 Pamplona, Spain
}

\begin{abstract}
We demonstrate a BOTDA sensor based on the use of the Brillouin phase-shift that performs measurements tolerant to non-local effects. This technique raises opportunities to increase the distance covered by these sensors and the maximum optical power of the probe wave injected to the fiber. As a result, the system has the potential to increase the SNR achieved at the last meters of the fiber. Proof-of-concept experiments demonstrate unaltered measurements of the phase-shift spectrum in a $20 \mathrm{Km}$ long fiber for large frequency-dependent distortions of the pump pulse.
\end{abstract}

Keywords: Stimulated Brillouin scattering, non-local effects, distributed fiber sensors, Brillouin phase-shift, BOTDA, nonlinear optics.

\section{INTRODUCTION}

Temperature and strain sensing of large area of coverage is a topic of major interest for several industrial sectors. In this context, Brillouin optical time domain analysis (BOTDA) sensors are considered a mature technology capable of monitoring these measurands over an extended distance range ${ }^{1}$ with high spatial resolution ${ }^{2}$.

However, as the monitoring distance increases, a limitation known as non-local effects is more insidious. The latter results from the accumulated energy transferred between the probe wave and the pump wave due to stimulated Brillouin scattering (SBS) along the sensing fiber. As a consequence, the pump pulse intensity is modified depending on the frequency difference between both waves leading to a distortion of the measurements. Therefore, the accuracy of the BOTDA sensor is impaired.

Different reports have focused on this effect trying to minimize it by building a theoretical model that quantifies the error in the determination of the Brillouin frequency shift (BFS) for different amounts of depletion ${ }^{3}$, by proposing a correction system based on an algorithm ${ }^{4}$, by evaluating its biasing effect ${ }^{5}$, by using a different interaction scheme between both waves $^{6}$ or simply by adjusting the probe wave power to avoid non-local effects at the measurement ${ }^{2}$. However, a key challenge faced by BOTDA sensors is to make measurements tolerable to distortions of the pump wave.

Recently, we proposed a BOTDA sensor based on a RF demodulation that improves the signal to noise ratio (SNR) in relation to the conventional direct detection scheme ${ }^{7}$. In addition, a phase-shift based BOTDA sensor intended for dynamic monitoring capable of performing measurements immune to the Brillouin gain or the probe wave optical power was demonstrated ${ }^{8}$. In this work, we propose an enhanced setup that is able to perform measurements tolerant to non-local effects. This allows an incrementing of the injected continuous wave (CW) optical power to enhance the SNR at the last position of the fiber.

\section{FUNDAMENTALS OF THE SYSTEM}

Fig. 1 schematically depicts the fundamentals of the proposed system. A phase-modulated probe wave is injected in one end of an optical fiber while a pump pulse is introduced at the other end. This probe wave interacts with the pump pulse via stimulated Brillouin scattering (SBS) and is directed to the receiver using a circulator. Considering that SBS interaction only affects the first sideband of the modulation, then the optical field at the input of the photodetector coming from the interaction of the pump and probe at a particular location in the fiber, $\mathrm{z}$, is given by the following expression:

$$
E(t)=-E_{S B} \exp \left(j 2 \pi\left(v_{0}-f_{R F}\right) t\right)+E_{0} \exp \left(j 2 \pi v_{0} t\right)+E_{S B} \exp \left(j 2 \pi\left(v_{0}+f_{R F}\right) t\right) H_{S B S}\left(v_{0}+f_{R F}, z\right)
$$

*javier.urricelqui@unavarra.es; phone 0034948 166046; fax 0034948 169720; unavarra.es 
where $E_{0}$ and $E_{S B}$ are the amplitudes of the optical fields of the carrier and first sidebands of the phase-modulated probe wave, $v_{0}$ is the optical frequency of the carrier, $f_{R F}$ is the modulation frequency and $H_{S B S}$ is the complex Brillouin loss spectrum at position $z$, which can be described by:

$$
H_{S B S}(v, z)=\exp \left(\frac{-g_{0} \Delta v_{B}}{\Delta v_{B}+2 j\left(v+v_{P}-v_{B}(z)\right)}\right)
$$

where $g_{0}$ is the local gain, $\Delta v_{B}$ is the Brillouin linewidth, $v_{B}$ is the Brillouin frequency shift at position $\mathrm{z}$ and $v_{P}$ is the optical frequency of the pump wave.
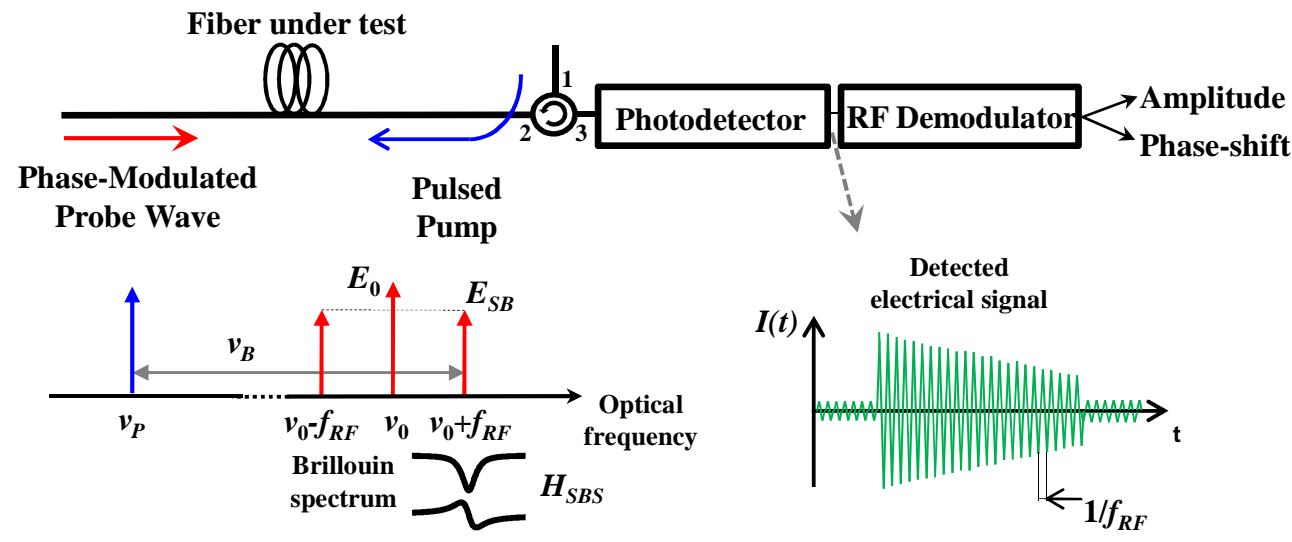

Figure 1.Schematic representation of SBS interaction and the received signal.

Detecting the optical field in Eq. 1, then the resultant RF signal can be written in phasorial form as:

$$
\left.I\right|_{f_{R F}}=R_{D} \sqrt{P_{0} P_{S B}}\left(H_{S B S}\left(v_{0}+f_{R F}, z\right)-1\right) \approx \frac{R_{D} g_{0} \sqrt{P_{0} P_{S B}} \Delta v_{B}}{\sqrt{\Delta v_{B}^{2}+4 \Delta v^{2}}} \exp \left(-j \arctan \left(2 \frac{\Delta v}{\Delta v_{B}}\right)\right)
$$

where $P_{0}$ and $P_{S B}$ are the optical powers of carrier and modulation sidebands, $R_{D}$ is the responsivity of the photodetector and $\Delta v=v_{0}+f_{R F}-v_{B}(z)+v_{P}$ is the detuning of the interacting sideband from the center of the Brillouin spectrum. The approximation for the last term has been obtained considering a small $g_{0}$. Notice in Eq. 3 that the detected RF phase-shift is independent of the particular local gain associated to SBS process, which is directly related to the amplitude of the pump pulse at that given location. This feature has major implications regarding the tolerance of the technique to non-local effects. The latter are produced due to the interaction of a pulsed pump wave counter-propagating to a $\mathrm{CW}$ probe wave, so that, at each location of the fiber, the energy transferred from probe wave to pump wave (in a loss-based BOTDA sensor) increases the intensity of the pump pulse. Therefore, the pump wave is amplified along its propagation through the sensing fiber. Moreover, as the SBS interaction depends on the frequency difference between both waves, then the resulting pump wave amplification is also frequency-dependent. This fact is shown in Fig. 2, where different amounts of non-local effects induced to the pump wave have been numerically calculated assuming a Brillouin linewidth of $30 \mathrm{MHz}$ and a uniform distribution of the BFS. This non-uniform frequency response of the pump wave leads to a distortion of the Brillouin loss spectrum. This is depicted in Fig. 3(a), where it has been assumed a worst case situation for non-local effects ${ }^{4}$, where the local BFS has been shifted by $\Delta v_{B} / 3$. This deformation of the Brillouin loss spectrum results in an impaired accuracy $\left(\Delta_{\mathrm{BFS}}\right)$ restricting the system performance. This fact is a major limitation in long range sensing. On the one hand, as the distance to be covered by such sensors is extended, the non-local effects induced to the pump wave increase due to the accumulated interaction along the optical fiber. On the other hand, as the probe wave optical power is increased, the energy transferred from the probe wave to the pump wave is also higher at each position of the fiber. This leads to a major distortion of the Brillouin loss spectrum for the same position. Consequently, the optical power of the probe wave must be maintained low, which in turn reduces the SNR at the final location of the sensing fiber. At last, the energy transferred between both waves depends on the whole BFS distribution along the sensing fiber. This results in greater non-local effects when the fiber has a uniform BFS distribution along the sensing fiber. 
In contrast to the conventional BOTDA, our technique is tolerant to non-local effects, as the detected phase-shift signal is not affected by the amplitude of the pump pulses. This is highlighted in Fig. 3(b), where the calculated RF phase-shift spectra remain unaltered for different amplifications of the pump pulse due to non-local effects. Therefore, our system is capable to increase the monitoring distance, the injected optical power and is independent of the BFS distribution along the fiber. As a consequence, the possibility to inject large optical probe powers to the fiber enables a rise in SNR enhancing the performance of the system. Notice that the SNR of our system is also increased due to the employment of the RF demodulation ${ }^{7}$ instead to the use of a conventional direct detection scheme. Therefore, our system enhances the SNR by two factors: the large probe wave optical powers injected to the fiber and the demodulation technique.

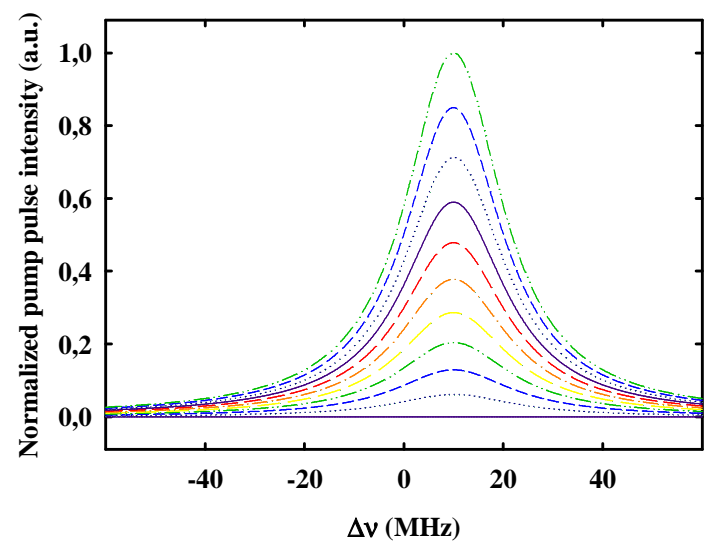

Figure 2. Normalized pump pulse intensity for different amounts of non-local effects.

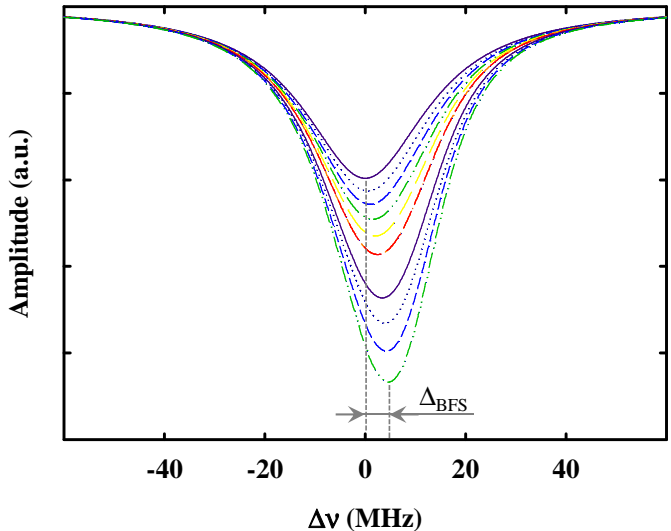

(a)

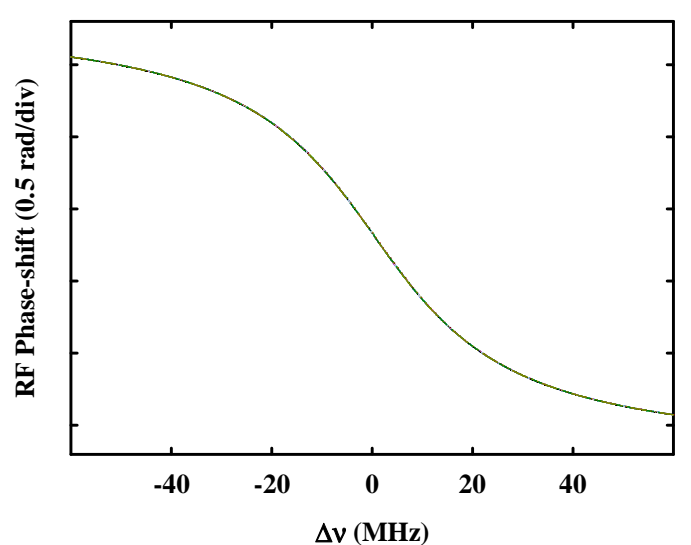

(b)

Figure 3. Calculated (a) amplitude spectra at conventional loss-based BOTDA and (b) RF phase-shift spectra of the proposed technique for different frequency responses of the pump gain.

\section{EXPERIMENTAL SETUP AND MEASUREMENTS}

The experimental setup shown in Fig. 4 was assembled in order to demonstrate the capabilities of the system. The output of a laser source is divided in two optical branches with an optical coupler. In the upper branch, the optical pump pulses are formed using a Mach-Zehnder electro-optic modulator (MZ-EOM) by the RF pulse-shaping technique so as to obtain clean and leakage-free pulses ${ }^{9}$. Then, one of the pump pulses is filtered by a fiber Bragg grating (FBG) to obtain a loss-based BOTDA system. The resultant pump pulse is amplified in an erbium doped fiber amplifier (EDFA) and its state of polarization is randomized with a polarization scrambler (PS) to reduce polarization-dependent effects. After that, it is directed via a circulator to a $20 \mathrm{Km}$-long fiber. In the lower branch, the probe wave is generated with an electro-optic phase modulator driven by a $850 \mathrm{MHz}$ RF signal. After interacting with the pump pulses via SBS, the probe signal is directed to a receiver and the resultant RF signal is demodulated. Finally, the BOTDA signal is captured in a digital oscilloscope. 


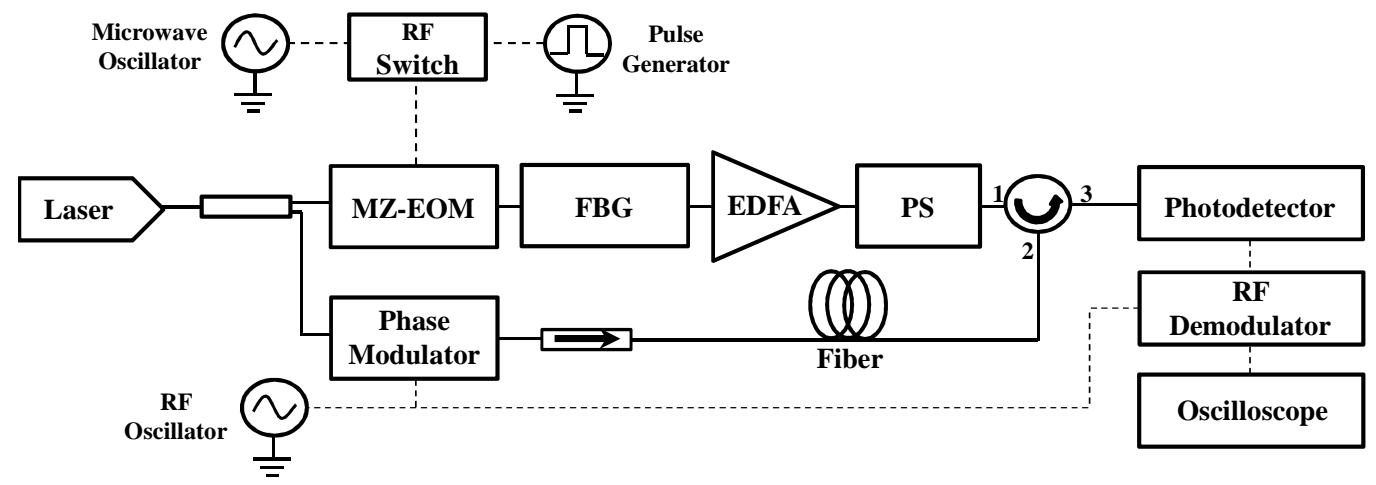

Figure 4.Experimental setup for the phase-shift-based BOTDA sensor.

In order to determine the amount of non-local effects induced at the proposed system, the pump pulse amplification resulting from the accumulated energy transferred from the probe wave to the pump wave was measured at the last location of the fiber. As this amount of non-local effects depends on the injected probe wave optical power, the pulse intensity was measured for two different probe wave optical powers. This is depicted in Fig. 5(a), where a 40\% increment of the pump pulse amplification is obtained for the larger optical probe power. Furthermore, as the accumulated energy transferred between the probe wave and the pump wave changes depending on the frequency difference between both waves, then the frequency response of the pump pulse intensity is non-uniform. This is clearly shown in Fig. 5 (b), where the energy of the pump pulse at each frequency separation between the probe wave and the pump wave was measured. In addition, the temporal response from the pump pulses at each frequency difference is also shown in Fig. 5(c).

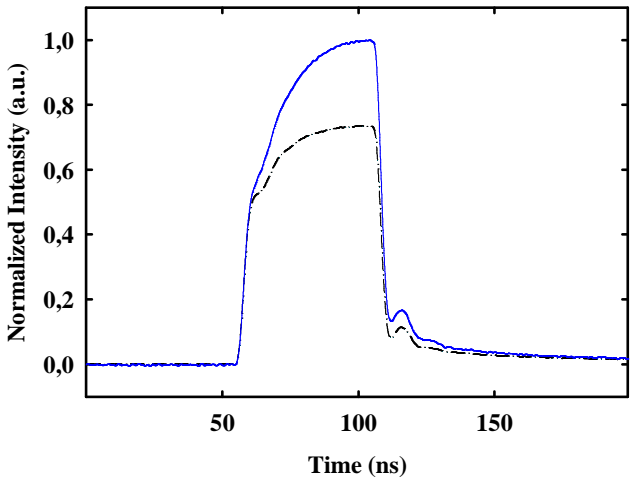

(a)

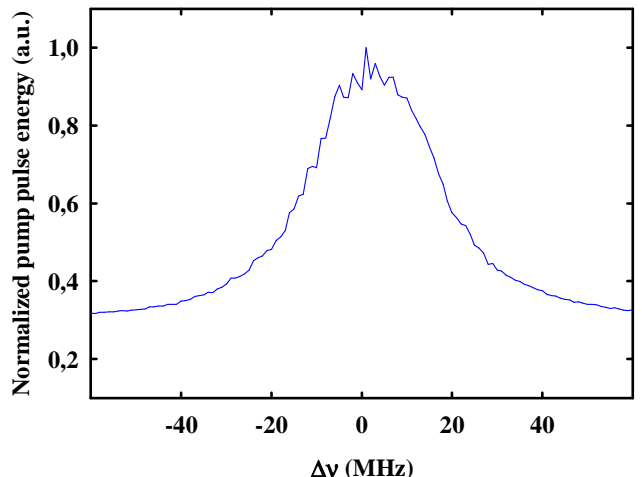

(b)

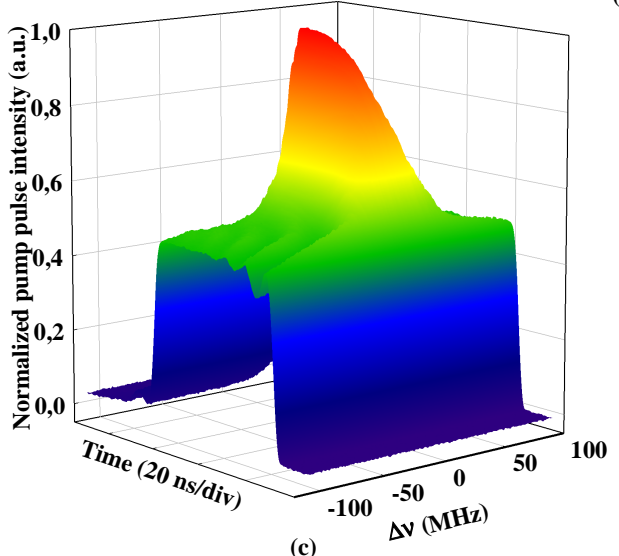

(c)

Figure 5. (a) Measured pulses for two probe wave optical powers (blue solid line $4.72 \mathrm{~mW}$ and black dashed line $2.61 \mathrm{~mW}$ ) at $\Delta v=0 \mathrm{MHz}$, (b) frequency response of the pulsed pump energy at the last location of the fiber and (c) measured pump pulses at each frequency difference between probe wave and pump wave for an optical probe power of $4.72 \mathrm{~mW}$. 
This increment in the pulsed pump intensity is clearly reflected in the amplitude of the BOTDA trace in Fig. 6 As it is shown, the amplitude of the resulting interaction between both waves differs from the typical attenuation suffered at the fiber $(0.2 \mathrm{~dB} / \mathrm{km})$.

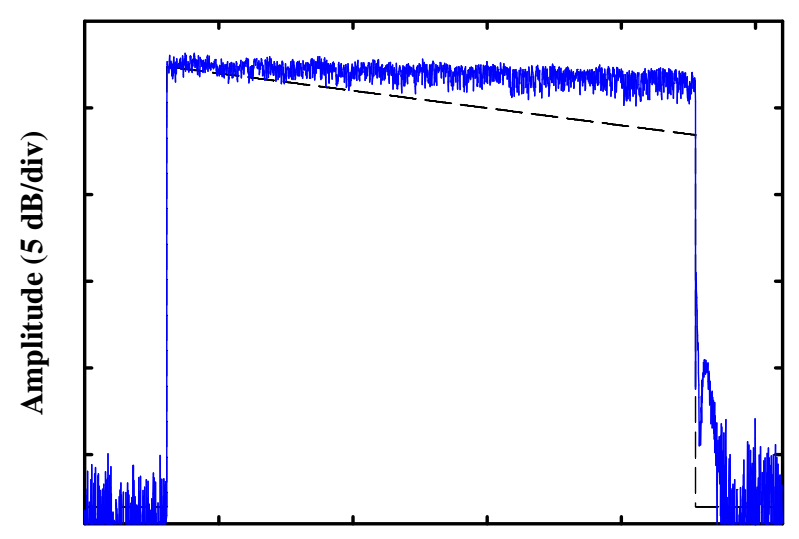

Distance $(5 \mathrm{Km} / \mathrm{div})$

Figure 6. Measured amplitude of the BOTDA trace (blue solid line) and theoretical BOTDA trace not affected by non-local effects and considering only attenuation in the fiber (black dashed line).

In addition, the RF phase-shift and amplitude spectra were measured for different probe wave optical powers at the last meters of interaction in the fiber. Fig. 7(a) shows that, indeed, the detected RF phase-shift remains unaltered while the amplitude (Fig. 7(b)) suffers the detrimental impact of non-local effects. Therefore, as the phase-shift spectrum is tolerant to non-local effects, then it is possible to inject higher probe wave optical powers to the sensing fiber in order to enhance the resultant SNR at the last position of the fiber.

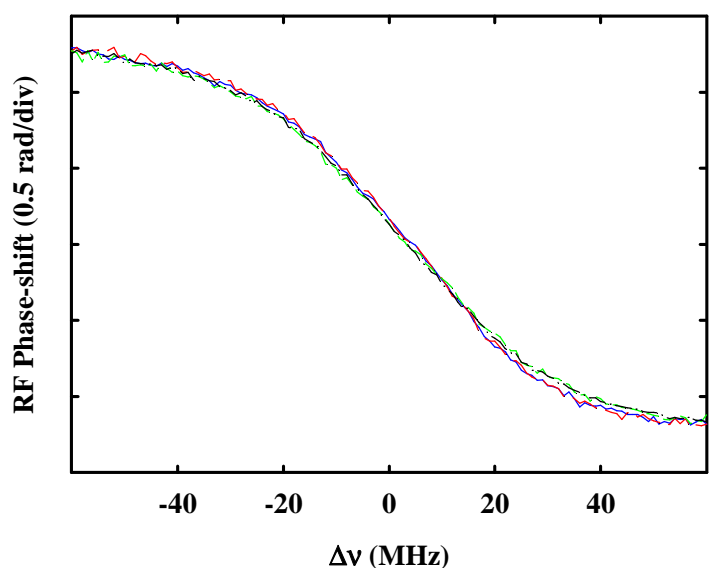

(a)

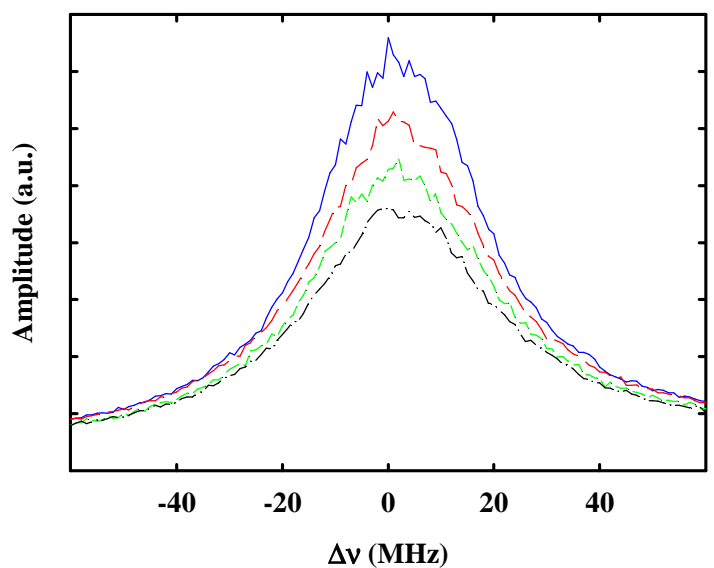

(b)

Figure 7. Scanned (a) phase-shift and (b) amplitude for different optical powers of the probe wave (blue solid line $4.72 \mathrm{~mW}$, red long dashed line $3.95 \mathrm{~mW}$, green short dashed line $3.29 \mathrm{~mW}$ and black dashed-dot line $2.61 \mathrm{~mW}$ ).

\section{CONCLUSION}

In this work we have presented a BOTDA sensor based on the use of the Brillouin phase-shift that is capable to perform measurements tolerant to non-local effects. The technique relies on the RF phase-shift spectrum, which is shown to be largely immune to factors that rise the non-local effects as: variations on the local gain, the distance to be covered by the system, the injected optical power of the probe wave and the distribution of the BFS along the fiber. Therefore, one of the fundamental drawbacks of the conventional BOTDA sensors is overcome. In addition, an experimental proof-of-concept has been performed with a $20 \mathrm{Km}$ long fiber obtaining identical RF phase-shift spectra for different 
amounts of depletion. Moreover, the results achieved illustrate that this system has potential to compensate the attenuation of the fiber suffered by the pump pulses providing BOTDA traces tolerant to the attenuation. Therefore, an increment of the SNR at the last position of the sensing fiber could be achieved.

\section{ACKNOWLEDGMENTS}

The authors wish to acknowledge financial support from the Spanish Ministerio de Educación y Ciencia through the project TEC2010-20224-C02-01 and from the Universidad Pública de Navarra.

\section{REFERENCES}

[1] Soto, M. A., Taki, M., Bolognini, G. and Di Pasquale, F., "Simplex-Coded BOTDA Sensor Over 120-km SMF With 1-m Spatial Resolution Assisted by Optimized Bidirectional Raman Amplification,"IEEE Photonic Tech. L. 24(20), 1823-1826 (2012).

[2] Angulo-Vinuesa, X., Martin-Lopez, S., Corredera, P. and Gonzalez-Herraez, M., "Raman-assisted Brillouin optical time-domain analysis with sub-meter resolution over $100 \mathrm{~km}$," Opt. Express 20(11), 12147-12154 (2012).

[3] Thévenaz, L., FoalengMafang, S., \& Lin, J. "Impact of pump depletion on the determination of the Brillouin gain frequency in distributed fiber sensors," Proc. SPIE 7753(2001).

[4] Minardo, A., Bernini, R., Zeni, L.,Thévenaz, L. and Briffod, F.,"A reconstruction technique for long-range stimulated Brillouin scattering distributed fibre-optic sensors: Experimental results," Meas. Sci. Technol. 16(4), 900-908 (2005).

[5] Dong, Y., Chen, L. and Bao, X., "System optimization of a long-range Brillouin-loss-based distributed fiber sensor," Appl. Opt. 49(27), 5020-5025 (2010).

[6] Bernini, R., Minardo, A., Zeni, L., "Long-range distributed Brillouin fiber sensors by use of an unbalanced double sideband probe," Opt. Express 19(24), 23845-23856 (2011).

[7] Zornoza, A., Sagues, M. and Loayssa, A., "Self-heterodyne detection for SNR improvement and distributed phase-shift measurements in BOTDA," J. Lightwave Technol. 30(8), 1066-1072 (2012).

[8] Urricelqui, J., Zornoza, A., Sagues, M., Loayssa, A., "Dynamic BOTDA measurements based on Brillouin phase-shift and RF demodulation," Opt. Express 20(24), 26942-26949 (2012).

[9] Zornoza, A., Olier, D., Sagues, M. and Loayssa, A., "Brillouin distributed sensor using RF shaping of pump pulses," Meas. Sci. Technol. 21(9), 094021 (2010). 
Copyright 2013 Society of Photo Optical Instrumentation Engineers. One print or electronic copy may be made for personal use only. Systematic re-production and distribution, duplication of any material in this paper for a fee or for commercial purposes, or modification of the content of the paper are prohibited. 